\title{
On Overlapping Divergences
}

\author{
Dirk KREIMER * \\ Dept. of Physics \\ Mainz Univ. \\ 55099 Mainz \\ Germany \\ MZ-TH/98-35, hep-th/9810022
}

\begin{abstract}
Using set-theoretic considerations, we show that the forest formula for overlapping divergences comes from the Hopf algebra of rooted trees.
\end{abstract}

\section{Motivation and Introduction}

The process of renormalization is governed by the forest formula, as derived for example in [1]. The underlying combinatorics is directly related to the Hopf algebra structure of rooted trees. This is evident in the case of Feynman diagrams which only provide nested or disjoint subdivergences. It is the purpose of this paper to show that the same Hopf algebra appears in the study of overlapping divergences. This was already shown using Schwinger Dyson equations [2], or by explicit considerations of divergent sectors [3], or differential equations on bare Green functions [4].

At such a level, one obtains a resolution of overlapping divergent graphs into a sum of rooted trees, to which then the combinatorics of the Hopf algebra of rooted trees applies [2, 国.

It was suggested to construct a Hopf algebra which directly considers overlapping divergent graphs, without using external input as Schwinger Dyson equations [5. However, as already mentioned in [4], this leads to the same Hopf algebra as for the case of non-overlapping divergences, as we will prove by set-theoretic considerations.

*Heisenberg Fellow, email: dirk.kreimer@uni-mainz.de 


\section{The Hopf algebra $\mathcal{H}_{R}$}

In this section we first repeat the definition of the Hopf algebra of decorated rooted trees, as it can be found in $\mid$. The rooted trees provide sets of vertices connected by edges. The vertices are labelled by decorations.

Each decoration corresponds to an analytic expression with a non-vanishing superficial degree of divergence, but free of subdivergences. Such analytic expressions are typically obtained from general Feynman graphs by shrinking superficially divergent subgraphs to a point. If for example $\Gamma$ is a superficially divergent Feynman graph which contains only one divergent subgraph $\gamma$, then one usually denotes by $\Gamma / \gamma$ the expression in which $\gamma$ is reduced to a point in $\Gamma$. When we speak of Feynman graphs in the following, this includes such quotients $\Gamma / \gamma$.

The Hopf algebra of decorated rooted trees, with vertices labelled by Feynman graphs free of subdivergences, is equivalent to the Hopf algebra on parenthesized words introduced in [2]. In the next section, we embark on some settheoretic considerations, which will prove useful in the study of overlapping divergences. In particular, we will assign a unique rooted tree to a set $M$ by imposing conditions on its subsets.

We follow section II of [4]. A rooted tree $t$ is a connected and simplyconnected set of oriented edges and vertices such that there is precisely one distinguished vertex which has no incoming edge. This vertex is called the root of $t$. Further, every edge connects two vertices and the fertility $f(v)$ of a vertex $v$ is the number of edges outgoing from $v$. The trees being simply-connected, each vertex apart from the root has a single incoming edge.

As in [4], we consider the (commutative) algebra of polynomials over $\mathbf{Q}$ in rooted trees, hence the multiplication $m\left(t, t^{\prime}\right)$ of two rooted trees means drawing them next to each other in arbitrary order.

Note that for any rooted tree $t$ with root $r$ we have $f(r)$ trees $t_{1}, \ldots, t_{f(r)}$ which are the trees attached to $r$. The unit element of this algebra is 1 , corresponding, as a rooted tree, to the empty set.

Let $B_{-}$be the operator which removes the root $r$ from a tree $t$ :

$$
B_{-}: t \rightarrow B_{-}(t)=t_{1} t_{2} \ldots t_{f(r)} .
$$

Fig.(11) gives an example.

Let $B_{+}$the operation which maps a monomial of $n$ rooted trees to a new rooted tree $t$ which has a root $r$ with fertility $f(r)=n$ which connects to the $n$ roots of $t_{1}, \ldots, t_{n}$.

$$
B_{+}: t_{1} \ldots t_{n} \rightarrow B_{+}\left(t_{1} \ldots t_{n}\right)=t .
$$

This is clearly the inverse to the action of $B_{-}$.

One has

$$
B_{+}\left(B_{-}(t)\right)=B_{-}\left(B_{+}(t)\right)=t
$$




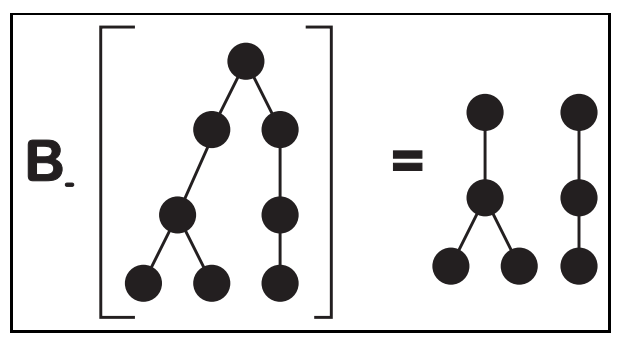

Figure 1: The action of $B_{-}$on a rooted tree.

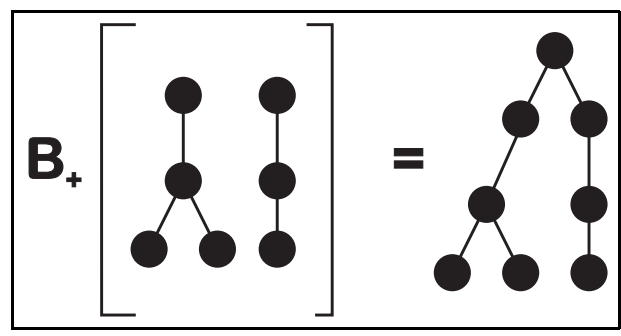

Figure 2: The action of $B_{+}$on a monomial of trees.

for any rooted tree $t$. Fig.(2) gives an example. For convenience, we define the rooted trees $t_{1}, t_{2}, t_{3_{1}}, t_{3_{2}}$ to be the trees with one, two or three vertices, given in Fig.(5) on the lhs from top to bottom.

We further set $B_{-}\left(t_{1}\right)=1, B_{+}(1)=t_{1}$.

We will introduce a Hopf algebra on such rooted trees by using the possibility to cut such trees in pieces. We start with the most elementary possibility. An elementary cut is a cut of a rooted tree at a single chosen edge, as indicated in Fig.(3). By such a cutting procedure, we will obtain the possibility to define a coproduct, as we can use the resulting pieces on either side of the coproduct.

But before doing so we finally introduce the notion of an admissible cut, also

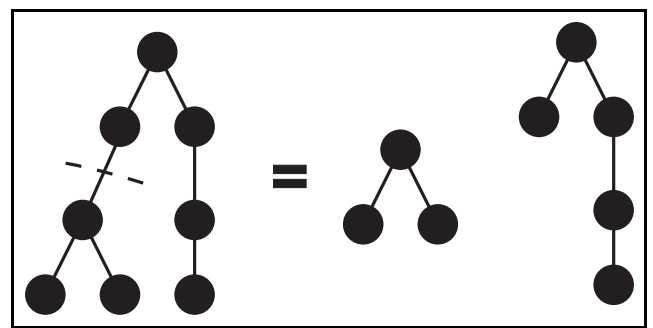

Figure 3: An elementary cut $c$ splits a rooted tree $t$ into two components, the fall-down $P^{c}(t)$ and the piece which is still connected to the root, $R^{c}(t)$. 


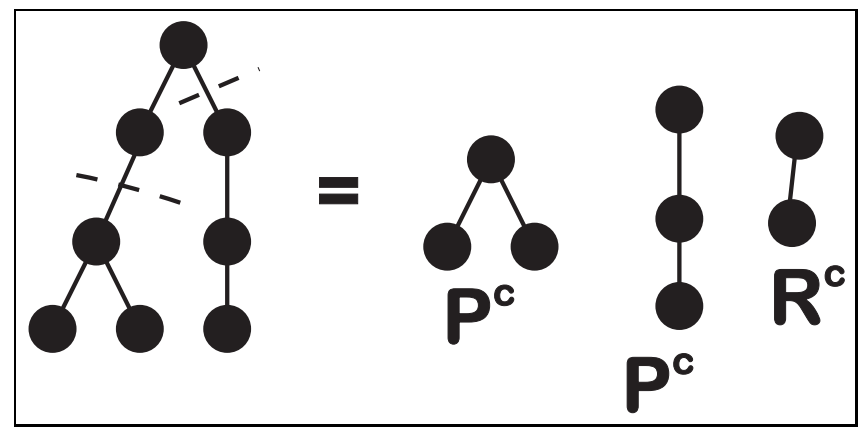

Figure 4: An admissible cut $C$ acting on a tree $t$. It produces a monomial of trees. One of the factors, $R^{C}(t)$, contains the root of $t$.

called a simple cut. It is any assignment of elementary cuts to a rooted tree $t$ such that any path from any vertex of the tree to the root has at most one elementary cut. Fig.(1) gives an example.

An admissible cut $C$ maps a tree to a monomial in trees. If the cut $C$ contains $n$ elementary cuts, it induces a map

$$
C: t \rightarrow C(t)=\prod_{i=1}^{n+1} t_{j_{i}} .
$$

Note that precisely one of these trees $t_{j_{i}}$ will contain the root of $t$. Let us denote this distinguished tree by $R^{C}(t)$. The monomial which is delivered by the $n-1$ other factors is denoted by $P^{C}(t)$.

The definitions of $C, P, R$ can be extended to monomials of trees in the obvious manner, by choosing a cut $C^{i}$ for every tree $t_{j_{i}}$ in the monomial:

$$
\begin{aligned}
C\left(t_{j_{1}} \ldots t_{j_{n}}\right) & :=C^{1}\left(t_{j_{1}}\right) \ldots C^{n}\left(t_{j_{n}}\right), \\
P^{C}\left(t_{j_{1}} \ldots t_{j_{n}}\right) & :=P^{C^{1}}\left(t_{j_{1}}\right) \ldots P^{C^{n}}\left(t_{j_{n}}\right), \\
R^{C}\left(t_{j_{1}} \ldots t_{j_{n}}\right) & :=R^{C^{1}}\left(t_{j_{1}}\right) \ldots R^{C^{n}}\left(t_{j_{n}}\right) .
\end{aligned}
$$

Let us now establish the Hopf algebra structure. Following [2, 14 we define the counit and the coproduct. The counit $\bar{e}: \mathcal{A} \rightarrow \mathbf{Q}$ is simple:

$$
\bar{e}(X)=0
$$

for any $X \neq 1$,

$$
\bar{e}(1)=1 \text {. }
$$

The coproduct $\Delta$ is defined by the equations

$$
\begin{aligned}
\Delta(1) & =1 \otimes 1 \\
\Delta\left(t_{1} \ldots t_{n}\right) & =\Delta\left(t_{1}\right) \ldots \Delta\left(t_{n}\right) \\
\Delta(t) & =t \otimes 1+\left(i d \otimes B_{+}\right)\left[\Delta\left(B_{-}(t)\right)\right],
\end{aligned}
$$




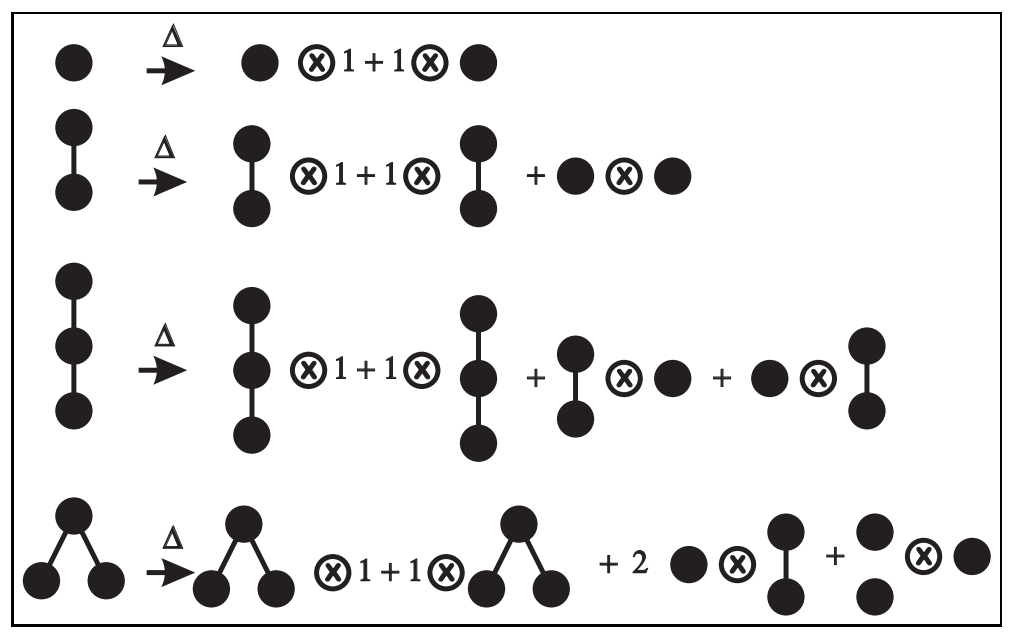

Figure 5: The coproduct. We work it out for the trees $t_{1}, t_{2}, t_{3_{1}}, t_{3_{2}}$, from top to bottom.

which defines the coproduct on trees with $n$ vertices iteratively through the coproduct on trees with a lesser number of vertices.

The coproduct can be written as [2, [1]

$$
\Delta(t)=1 \otimes t+t \otimes 1+\sum_{\text {adm. cuts } C \text { of } t} P^{C}(t) \otimes R^{C}(t) .
$$

Up to now we have established a bialgebra structure. It is actually a Hopf algebra. Following [2, 4] we find the antipode $S$ as

$$
\begin{aligned}
S(1) & =1 \\
S(t) & =-t-\sum_{\text {adm. cuts } C \text { of } t} S\left[P^{C}(t)\right] R^{C}(t) .
\end{aligned}
$$

Let us give yet another formula to write the antipode, which one easily derives using induction on the number of vertices [2, 此:

$$
S(t)=-\sum_{\text {all cuts } C \text { of } t}(-1)^{n_{C}} P^{C}(t) R^{C}(t),
$$

where $n_{C}$ is the number of single cuts in $C$.

This time, we have a non-recursive expression, summing over all cuts $C$, relaxing the restriction to admissible cuts.

By now we have established a Hopf algebra on rooted trees, using the set of rooted trees, the commutative multiplication $m$ for elements of this set, the unit 1 and counit $\bar{e}$, the coproduct $\Delta$ and antipode $S$. We call this Hopf algebra $\mathcal{H}_{R}$. 
Still following [2, 4] we allow to label the vertices of rooted trees by Feynman graphs without subdivergences, in the sense described before.

Quite general, if $Y$ is a set of primitive elements providing labels, we call the resulting Hopf algebra $\mathcal{H}_{R}(Y)$. Let us also mention that

$$
m[(S \otimes i d) \Delta(t)]=\bar{e}(t)=0=\sum S\left(t_{(1)}\right) t_{(2)},
$$

where we introduced Sweedler's notation $\Delta(t)=: \sum t_{(1)} \otimes t_{(2)}$, and $i d$ is the identity map $\mathcal{H}_{R} \rightarrow \mathcal{H}_{R}$.

We finally note the following definition: for a rooted tree $t$ let $n_{v}(t)$ be the number of its vertices. This extends to a monomial of rooted trees in the obvious manner, $n_{v}\left(\prod_{i} T_{i}\right)=\sum_{i} n_{v}\left(T_{i}\right)$.

Ultimately, we work in the vector space of finite linear combinations of monomials in rooted trees. Hence for such a linear combination $T:=\sum_{i} q^{i} X_{i}, i \in \mathbf{I}$, for some index set $\mathbf{I}$, we define

$$
n_{v}(T):=\max \left\{n_{v}\left(X_{i}\right) \mid i \in \mathbf{I}\right\} .
$$

\section{A set theoretic approach}

\section{$2.1 \quad$ Notation}

Let $\#(M)$ be the cardinality of any set $M$.

For any given finite set $M$ we let $\mathcal{P}(M)$ be the set of all proper subsets of $M$. Further, we let

$$
\mathcal{P}_{X}(M) \subset \mathcal{P}(M)
$$

be the set of all proper subsets of $M$ which fulfill the condition $X$. Thus, if $\mathbf{X}$ is the boolean operator which is true when the condition $X$ is satisfied, we have

$$
\mathcal{P}_{X}(M)=\{\gamma \in \mathcal{P}(M) \mid \mathbf{X}(\gamma)\} .
$$

If we impose no condition we write $X=\emptyset$, hence $\mathcal{P}_{\emptyset}(M) \equiv \mathcal{P}(M)$. If we want to stress that a subset $\gamma \subset M$ fulfills condition $X$, we write $\gamma_{X}^{\subset} M$.

Let $\gamma_{i}, \gamma_{j} \subset \mathcal{P}(M), i \neq j$, be two elements of $\mathcal{P}_{X}(M)$, hence two subsets of $M$. If

$$
\gamma_{i} \cap \gamma_{j}=\emptyset
$$

we call $\gamma_{i}, \gamma_{j}$ disjoint.

Else, if

$$
\gamma_{i} \subset \gamma_{j} \text { or } \gamma_{j} \subset \gamma_{i}
$$

we call $\gamma_{i}, \gamma_{j}$ nested.

Finally, if $\gamma_{i}, \gamma_{j}$ are neither disjoint nor nested, we call them overlapping. They then have a nontrivial intersection $U:=\gamma_{i} \cap \gamma_{j} \neq \emptyset$, which is a proper subset of 
each, $\gamma_{i} \supset U \subset \gamma_{j}$.

If $\gamma_{i}, \gamma_{j}$ are not overlapping, we call them tree-related, for reasons which become obvious in a moment.

For a given set $X$ of mutually tree-related sets $\gamma_{i}$, we say that another set $\gamma$ is overlapping with $X$ if $\gamma$ is overlapping with at least one element of $X$.

If a set $\gamma \subset \mathcal{P}_{X}(M)$ can be written as a union of mutually disjoint sets $\gamma_{i} \subset \mathcal{P}_{X}(M)$,

$$
\gamma=\cup_{i \in \mathbf{I}} \gamma_{i}
$$

for some index set $\mathbf{I}$, we say that $\gamma$ is reducible. Otherwise, we say it is irreducible (w.r.t. $X$ ). Note that reducibility depends on the chosen condition $X$.

Let $M / \gamma$ denote the complement of the set $\gamma \subset M$ with respect to $M$,

$$
M=M / \gamma \cup \gamma .
$$

\section{$2.2 \quad$ Basic Results}

It is our task to find all elements $p \in \mathcal{P}\left(\mathcal{P}_{X}(M)\right)$ which fulfill the following three conditions

i) $p$ consists of mutually tree-related sets $\in \mathcal{P}_{X}(M)$,

ii) all elements of $p$ are irreducible,

iii) $p$ is complete: for all $\gamma \subset \mathcal{P}_{X}(M)$ such that $\gamma \notin p \Leftrightarrow \gamma$ is overlapping with

$p$.

For an irreducible $M$, let the set of all such $p$, that is the set of all complete, irreducible, tree-ordered elements of $\mathcal{P}\left(\mathcal{P}_{X}(M)\right)$ be denoted by $\mathcal{P}_{X}^{\text {cit }}(M)$.

Prop.1 To each such $p \in \mathcal{P}_{X}^{\text {cit }}(M)$, we can assign a rooted tree $T_{X}(p)$ with $n=(\#(p)+1)$ vertices.

Proof: We draw $n$ points in the plane, which furnish the set of vertices of the rooted tree. To one of these points, we associate the set $M$. It will become the root. To each of the other $n-1$ points we associate one element of $p$. Let $v\left(\gamma_{i}\right)$ denote the vertex which is labelled by the set $\gamma_{i} \in p$ in this process.

Now we can construct the edges. For that, we connect two vertices $v\left(\gamma_{i}\right), v\left(\gamma_{k}\right)$ by an edge pointing from $v\left(\gamma_{k}\right)$ to $v\left(\gamma_{i}\right)$ if and only if the following two conditions are fulfilled:

i) $\gamma_{i} \subset \gamma_{k}$,

ii) there is no further set $\gamma_{j} \in p$ such that $\gamma_{i} \subset \gamma_{j} \subset \gamma_{k}$.

Here, we allow $\gamma_{k}$ to be the set $M$ itself: $\gamma_{k} \in\{p \cup M\}$.

The resulting tree is simply-connected, due to the fact that all elements of $p$ are mutually tree-ordered. Further, it has a distinguished root.

For a chosen vertex $v$ of a rooted tree $T_{X}(p)$ let $\gamma(v)$ be the set associated to that vertex. Further, assume that $f(v)=k$, hence $v$ connects via $k$ outgoing edges to vertices $v_{1}, \ldots, v_{k}$, say. The corresponding sets $\gamma\left(v_{1}\right), \ldots, \gamma\left(v_{k}\right)$ are necessarily mutually disjoint, as $T_{X}(p)$ is simply-connected. 
Define

$$
\gamma_{v}:=\cup_{i:=1}^{f(v)} \gamma\left(v_{i}\right)
$$

Prop.2 $\mathcal{P}_{X}\left(\gamma(v) / \gamma_{v}\right)=\emptyset$.

Proof: $\gamma(v)$ is irreducible as it is an element of $p$. Hence $\gamma(v) / \gamma_{v} \neq \emptyset$. By definition, $\gamma_{v}$ is the union of all sets $\gamma\left(v_{i}\right) \in p$ which are subsets $\gamma\left(v_{i}\right) \subset \gamma(v)$. If there would be an element $\gamma^{\prime}$ in $\mathcal{P}_{X}\left(\gamma(v) / \gamma_{v}\right)$, this would imply that $\gamma^{\prime}$ is a non-overlapping subset of $\gamma(v)$ which is not in $\gamma_{v}$. Contradiction.

The linear combination $T_{X}(M)$ assigned to the irreducible set $M$ is the sum

$$
T_{X}(M):=\sum_{p \in \mathcal{P}_{X}^{c i t}(M)} T_{X}(p) .
$$

For a reducible $M$ we can write $M=\cup_{i} M_{i}$ for some mutually disjoint irreducible sets $M_{i}$. We then set $T_{X}(M)=\sum_{i} T_{X}\left(M_{i}\right)$.

An example might be in order. Let $M=\{a, b, c\}$. First, choose $X=\emptyset$. All subsets which contain more than one element are reducible. Thus, $T_{X}(M)$ is the product $t_{1}(a) t_{1}(b) t_{1}(c)$ of three disjoint roots, labelled $\{a\},\{b\},\{c\}$.

Next, let $X$ be the condition that $a$ is contained in the subset but not $c$. Then, $\{a\}$ and $\{a, b\}$ are irreducible proper subsets. $\mathcal{P}_{X}^{c i t}(M)$ contains a single set $p=\{\{a\},\{a, b\}\}$, and we obtain $T_{X}(p)=t_{3_{1}}$ (see Fig.(5)) with the set $M$ labelling the root, which is connected to a vertex labelled by $\{a, b\}$, and finally this vertex is connected to a third one labelled by $\{a\}$.

Finally, choose $X$ to be the condition that $a$ is contained in the subset. Then, $\{a\},\{a, b\},\{a, c\}$ are irreducible proper subsets. The latter two are overlapping. $\mathcal{P}_{X}^{\text {cit }}(M)$ consists of two elements $p_{1}, p_{2}$, say, where $p_{1}=\{\{a\},\{a, b\}\}$ and $p_{2}=$ $\{\{a\},\{a, c\}\} . T_{X}\left(p_{1}\right)$ and $T_{X}\left(p_{2}\right)$ both realize $t_{3_{1}}$ with appropriate decorations. Consider Fig.(6) for a visualization of these examples.

\subsection{The Hopf algebra structure of $\mathcal{P}_{X}(M)$}

To each set $M$ we can assign a depth $d_{X}(M)$ as $d_{X}(M)=n_{v}\left(T_{X}(M)\right)$, according to (12). This gives us a decomposition on the set $\mathcal{M}$ of all irreducible (w.r.t.X) finite sets through the grading by depth,

$$
\mathcal{M}=\mathcal{M}^{[1]} \cup \mathcal{M}^{[2]} \cup \ldots \cup \mathcal{M}[k] \cup \ldots,
$$

which obviously depends on the condition $X$. Here, $\mathcal{M}^{[1]}$ are all sets $M$ which have no proper subset which fulfills condition $X$, hence of depth $d_{X}(M)=1$, $\mathcal{M}^{[2]}$ are all sets of depth two, such that all their proper subsets which fulfill $X$ are from $\mathcal{M}^{[1]}$, and in general $\mathcal{M}^{[k]}$ contains all sets of depth $k$, and hence has proper subsets of depth $\leq k-1$.

\footnotetext{
${ }^{1}$ If there is more than one possibility to write $M$ as a union of disjoint sets $M_{i}$, we sum over all trees which we obtain from the consideration of all possibilities how to decompose $M$ into these various disjoint subsets. We will not meet this case in this paper, though.
} 


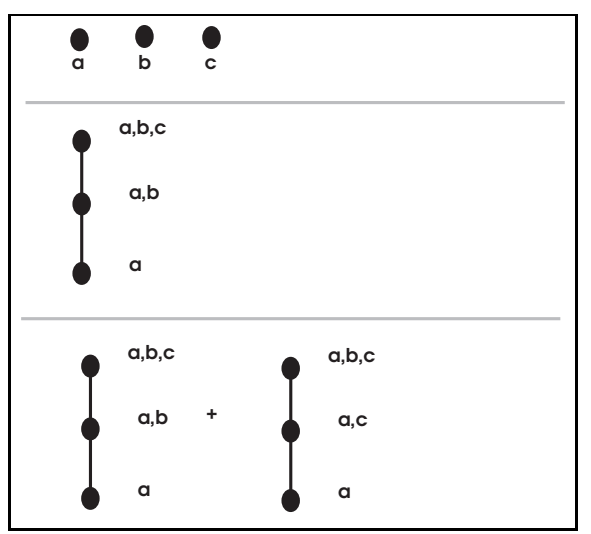

Figure 6: The examples show how various $T_{X}(\{a, b, c\})$ are generated by different conditions $X$. From top to bottom, we have i) $X=\emptyset$, ii) $X: a \in \gamma, c \notin \gamma$, iii) $X: a \in \gamma$.

We want to establish a Hopf algebra of rooted trees on $\mathcal{M}$. For this, it is sufficient to study irreducible sets $M$. We will take elements of $\mathcal{M}^{[1]}$ as primitive elements. By definition, $T(M)=t_{1}(M)$ for $M \in \mathcal{M}^{[1]}$, which justifies this choice.

We call a set $M$ non-overlapping, if $\mathcal{P}_{X}(M)$ is tree-ordered, hence if all its subsets which fulfill $X$ are tree related amongst each other.

Prop.3 If $M$ is non-overlapping, $\#\left(\mathcal{P}_{X}^{\text {cit }}(M)\right)=1$.

Proof: All elements of $\mathcal{P}_{X}(M)$ can be tree-ordered amongst themselves by assumption. As any element $p \in \mathcal{P}_{X}^{c i t}(M)$ is complete and contained in $\mathcal{P}_{X}(M)$, there can be only one such element $p$.

Two final definitions: If $X$ is a given condition,

$$
\mathcal{P}_{X}(M)=\{u \in \mathcal{P}(M) \mid \mathbf{X}(u)\},
$$

then, for $\gamma \in \mathcal{P}_{X}(M), X_{\gamma}$ is defined to be the condition

$$
\mathcal{P}_{X_{\gamma}}(M)=\left\{u \in \mathcal{P}(M) \mid \mathbf{X}(u) \text { and } u \notin\left\{\gamma \cup \mathcal{P}_{X}(\gamma)\right\}\right\} .
$$

We call a condition $X$ an orderly condition if and only if $T_{X_{\gamma}}(M)=T_{X}(M / \gamma)$, $\forall \gamma \in \mathcal{P}_{X}(M)$. This means that checking the condition $X$ and then eliminating all elements of $\mathcal{P}_{X}(M)$ which belong as well to $\gamma \cup \mathcal{P}_{X}(\gamma)$ is the same as first eliminating $\gamma$ and checking the condition $X$ on the reduced set $M / \gamma$.

Let us give an example of an orderly condition.

Example: Consider a space $Y$ and a set $\sigma_{Y}$ of subsets of $Y$. Endow $Y$ with the topology generated by $\sigma_{Y}$ as a subbasis.] Endow any space $Y / \gamma, \gamma \in \sigma_{Y}$ with

\footnotetext{
${ }^{2}$ Any set $\sigma$ of subsets of a space generates a topology. The open sets are unions of finitely many intersections of elements of $\sigma$, and $\sigma$ is the subbasis of this topology.
} 
its induced topology, which is generated by the subbasis $\left\{u / \gamma \mid u \in \sigma_{Y}\right\}$. Let $X$ be the condition that a subset $\gamma \subset Y$ must fulfill $\gamma \in \sigma_{Y}$ to be in $\mathcal{P}_{X}(Y)$. Then, $X$ is an orderly condition. Indeed, $T_{X}(Y / \gamma)$ is the forest $T_{X}(Y)$ in which all vertices decorated by $\gamma$ or its subsets in $\mathcal{P}_{X}(\gamma)$ are deleted, and so is $T_{X_{\gamma}}(Y)$.

On the other hand, note that the examples in Fig.(6) give non-orderly conditions for $X \neq \emptyset$.

We want to establish a Hopf algebra of rooted trees $\mathcal{H}_{R}\left(\mathcal{M}^{[1]} \cup_{i=2}^{\infty} U^{[i]}\right)$ which assigns to each $M \in \mathcal{M}^{[k]}$ a sum of rooted trees $T_{M}$ such that its coproduct takes the form

$$
\Delta\left(T_{M}\right)=\sum_{\gamma \underset{X}{\subset} M} T_{\gamma} \otimes T_{M / \gamma}
$$

The sum is over all subsets $\gamma \subset M$ such that $\gamma$ fulfills condition $X$. We do not demand that $\gamma$ is irreducible. It is thus allowed that $\gamma$ is the union of disjoint sets $\gamma_{i}$ which themselves fulfill condition $X$ and are irreducible.

The notation $\cup_{i=2}^{\infty} U^{[k]}$ refers to the iterative manner in which we will achieve our goal. To achieve our goal for sets $M$ of depth one is trivial. We take $\mathcal{M}^{[1]}$ as the set of decorations for $\mathcal{H}_{R}$ and are done. Next, we will construct a set of decorations, $U^{[2]}$ such that $\mathcal{H}_{R}\left(\mathcal{M}^{[1]} \cup U^{[2]}\right)$ achieves the desired goal for all sets of depth up to two. Then, we further enlarge this set by $U^{[3]}$ so that the coproduct in $\mathcal{H}_{R}\left(\mathcal{M}^{[1]} \cup U^{[2]} \cup U^{[3]}\right)$ agrees with (13) for sets $M$ of depth up to three and so on. In general, we show that if one has succeeded at depth $k$ that there is a set of decorations $U^{[k+1]}$ which are primitive under the coproduct of $\mathcal{H}_{R}$, such that one obtains the desired form (13).

It will turn out that $T_{M}$ is a sum of rooted trees containing $T_{X}(M)$. Further, $\left[T_{M}-T_{X}(M)\right]$ is a sum of rooted trees which fulfills $n_{v}\left(T_{M}-T_{X}(M)\right)<$ $n_{v}\left(T_{X}(M)\right)$.

For non-overlapping sets $M$, there is an immediate natural Hopf algebra structure $\mathcal{H}_{R}\left(\mathcal{M}^{[1]}\right)$. It is natural in the sense that the coproduct assumes the form $(13)$ :

Prop.4 For non-overlapping sets $M$ we have

$$
\Delta\left(T_{X}(M)\right)=\sum_{\gamma_{X}^{\subset} M} T_{X}(\gamma) \otimes T_{X_{\gamma}}(M) .
$$

Proof: For non-overlapping sets $M, T_{X}(M)$ is a single rooted tree $T_{X}(M)=$ $T_{X}(p)$. Admissible cuts on this rooted tree and subsets $\gamma$ in the sum are in one-to-one correspondence, by construction. Let $\gamma_{C}$ be the set corresponding to the chosen admissible cut $C$. By the definition of $T_{X}(\gamma), T_{X}(\gamma)=P^{C}\left(T_{X}(M)\right)$. Further, $R^{C}\left(T_{X}(M)\right)$ is the decorated tree which remains connected with the root under the admissible cut. By definition of $X_{\gamma}, T_{X_{\gamma}}(M)=R^{C}\left(T_{X}(M)\right)$ as both rooted trees are obtained from $T_{X}(M)$ by eliminating all vertices and edges corresponding to $T_{X}(\gamma)$. Further, by Prop.2 we can decorate the rooted tree $T_{X}(M)$ with elements from $\mathcal{M}^{[1]}$. 
Note that for an orderly condition $X,(14)$ takes the form

$$
\Delta\left(T_{X}(M)\right)=\sum_{\gamma_{X}^{\subset} M} T_{X}(\gamma) \otimes T_{X}(M / \gamma) .
$$

Hence we set

$$
T_{\gamma}=T_{X}(\gamma), T_{M / \gamma}=T_{X}(M / \gamma),
$$

to obtain the desired form (13) for all non-overlapping $M \in \mathcal{M}^{\text {nol }}$, the set of all sets $M$ which are non-overlapping. This is consistent as if $M$ is non-overlapping, so are all elements in $\mathcal{P}_{X}(M)$.

To simplify notation, let us assume in the following that $X$ is an orderly condition. When we come to Feynman graphs in the next section, we will actually find the relevant condition $X$ to be an orderly condition. However, the general case demands not much more than a replacement $T_{X}(M / \gamma) \rightarrow T_{X_{\gamma}}(M)$ and a slightly refined decomposition of $\mathcal{M}$.

So far, we found that all elements in $\mathcal{M}^{\text {nol }}$ have the desired form. From now on let $\Delta_{1}$ be the coproduct of $\mathcal{H}_{R}\left(\mathcal{M}^{[1]}\right)$. We have just shown that it has the desired form on $\mathcal{M}^{\text {nol }}$. We stress that $\Delta_{1}$ is defined on all rooted trees with decorations in $\mathcal{M}^{[1]}$.

We now want to show that for the other elements, which are overlapping sets $M$, we can find a Hopf algebra of rooted trees with a coproduct which has the desired form (13), by simply adding more decorations. As an aside, we will gain a systematic decomposition into primitive elements, which corresponds to a skeleton expansion at the level of QFT, as we will see later on.

We will proceed by induction on the depth. There are no overlapping sets $M$ in $\mathcal{M}^{[1]}, \mathcal{M}^{[1]} \subset \mathcal{M}^{\text {nol }}$.

Hence we start the induction by considering sets in $\mathcal{M}^{[2]}$. We want to construct a Hopf algebra of rooted trees $\mathcal{H}_{R}\left(\mathcal{M}^{[1]} \cup U^{[2]}\right)$ such that its coproduct $\Delta_{2}$ again can be written in the form (13). $U^{[2]}$ is a set of decorations, hence we demand $\Delta_{2}(u)=u \otimes 1+1 \otimes u, \forall u \in U^{2]}$.

Let $M \in \mathcal{M}^{[2]}$ be irreducible and overlapping. Then, each $p \in \mathcal{P}_{X}^{[c i t]}(M)$ is in $\mathcal{M}^{[1]}$.

Let us assign to $M$ an element $T_{M}$ and set

$$
\Delta_{2}\left(T_{M}\right)=T_{M} \otimes 1+1 \otimes T_{M}+\sum_{\gamma_{X}^{\subset} M} T_{\gamma} \otimes T_{M / \gamma}
$$

Due to the definition of $\mathcal{P}_{X}^{\text {cit }}(M)$ this can be written as

$$
\Delta_{2}\left(T_{M}\right)=T_{M} \otimes 1+1 \otimes T_{M}+\sum_{p \in \mathcal{P}_{X}^{\text {cit }}(M)} T_{p} \otimes T_{M / p} .
$$

But $p \in \mathcal{M}^{[1]}$ and $M / p \in \mathcal{M}^{[1]}$, hence $T_{p}=T_{X}(p), T_{M / p}=T_{X}(M / p)$, by (16). 
Also, the coproduct $\Delta_{1}$ of $\mathcal{H}_{R}\left(\mathcal{M}^{[1]}\right)$ is defined on the sum of rooted trees $T_{X}(M)$ and reads

$$
\Delta_{1}\left(T_{X}(M)\right)=T_{X}(M) \otimes 1+1 \otimes T_{X}(M)+\sum_{p \in \mathcal{P}_{X}^{\text {cit }}(M)} T_{X}(p) \otimes T_{X}(M / p) .
$$

Thus, we find that, for $U_{M}:=T_{M}-T_{X}(M)$,

$$
\Delta_{2}\left(U_{M}\right)=U_{M} \otimes 1+1 \otimes U_{M}
$$

$U_{M}$ reveals itself to be a primitive element with respect to $\Delta_{2}$. This suggests to define $U^{[2]}$ via the union of all elements $U_{M}=T_{M}-T_{X}(M)$ where $M$ is of depth two and overlapping. As $U_{M}$ is primitive we identify it with a decoration $u_{M}$ of the tree $t_{1}, t_{1}\left(u_{M}\right)=U_{M}$ and obtain

$$
U^{[2]}=\left\{u_{M} \mid t_{1}\left(u_{M}\right)=T_{M}-T_{X}(M), M \in \mathcal{M}^{[2]} \wedge M \notin \mathcal{M}^{\text {nol }}\right\}
$$

Hence we find that $\Delta_{2}$ is the coproduct of the Hopf algebra of rooted trees

$$
\mathcal{H}_{R}\left(\mathcal{M}^{[1]} \cup U^{[2]}\right),
$$

where $U^{[2]}$ is the set of decorations corresponding to primitive elements $U_{M} \equiv$ $T_{M}-T_{X}(M), M$ being an overlapping set in $\mathcal{M}^{[2]}$. The primitive elements of this Hopf algebra are $t_{1}(M), M \in \mathcal{M}^{[1]}$ and the elements $U_{M}$ defined above.

Note that the element $T_{M}$ is resolved into the linear combination of trees $T_{M}=T_{X}(M)+t_{1}\left(u_{M}\right)$, as desired. Note further that we can write the coproduct $\Delta_{2}$ as

$$
\Delta_{2}\left(T_{M}\right)=T_{M} \otimes 1+1 \otimes T_{M}+(i d-E \circ \bar{e}) \otimes(i d-E \circ \bar{e}) \Delta_{1}\left(T_{X}(M)\right),
$$

where $E: \mathbf{Q} \rightarrow \mathcal{H}_{R}$ is given by $E(q)=q 1$. Obviously we left the counit $\bar{e}$ unchanged, $\bar{e}(1)=1, \bar{e}(T)=0, \forall T \neq 1$.

At this point the attentive reader might ask why we not simply set $T_{M}=$ $T_{X}(M)$, as this would still deliver the natural form (13). But our point is to show that any attempt to find a Hopf algebra which has the natural form (13) will be a Hopf algebra of rooted trees, with an appropriate set of primitive elements. This completely puts the combinatorical problem of renormalization at rest and settles its algebraic structure as determined by the Hopf algebra structure of rooted trees, which, fascinatingly, not only describes renormalization but also the combinatorics of the diffeomorphism group [1].

Let us continue then. Thus, let $M \in \mathcal{M}^{[k]}$ be irreducible and overlapping. Assume we found a Hopf algebra of rooted trees $\mathcal{H}_{R}\left(\mathcal{M}^{[1]} \cup_{i=2}^{k} U^{[i]}\right)$ with coproduct $\Delta_{k}$ such that in this Hopf algebra there is a linear combination $T_{M}$ of elements such that the coproduct obtains the form (13),

$$
\Delta_{k}\left(T_{M}\right)=T_{M} \otimes e+e \otimes T_{M}+\sum_{\gamma_{X}^{\subset} M} T_{\gamma} \otimes T_{M / \gamma}
$$


We want to induce that the same holds for $M_{+} \in \mathcal{M}^{[k+1]}$.

Let $\gamma_{X}^{\subset} M_{+}$be given, and let $M_{+} \in \mathcal{M}^{[k+1]}$ be overlapping. Then, consider all the terms in

$$
\Delta_{1}\left[T_{X}\left(M_{+}\right)\right]=\sum_{p \in \mathcal{P}_{X}^{\text {cit }}\left(M_{+}\right)}\left[\sum_{\text {adm.cts. }} C_{C^{p} \text { of } T_{X}(p)} P^{C(p)}\left[T_{X}(p)\right] \otimes R^{C(p)}\left[T_{X}(p)\right]\right],
$$

which correspond to the set $\gamma$. This is well-defined: any two overlapping sets $\gamma, \gamma^{\prime} \in \mathcal{P}_{X}\left(M_{+}\right)$will correspond to branches of different trees $T_{X}(p), T_{X}\left(p^{\prime}\right)$, as elements $p, p^{\prime}$ are tree-ordered. Further, each single elementary cut corresponds to some subset $\gamma_{X}^{\subset} M_{+}$. We can thus organize the above sum in groups of terms corresponding to $\gamma_{X}^{\subset} M_{+}$. Finally, the completeness of all elements of $\mathcal{P}_{X}^{\text {cit }}\left(M_{+}\right)$ guarantees that all admissible cuts which correspond to $\gamma$ conspire to give $T_{X}(\gamma)$, and all terms on the other side of the tensorproduct conspire to give $T_{X}\left(M_{+} / \gamma\right)$, for an orderly condition $X$. We get

$$
\begin{aligned}
\Delta_{1}\left[T_{X}\left(M_{+}\right)\right] & =\sum_{p \in \mathcal{P}_{X}^{\text {cit }}\left(M_{+}\right)}\left[\sum_{\text {adm.cts. } C^{p} \text { of } T_{X}(p)} P^{C(p)}\left[T_{X}(p)\right] \otimes R^{C(p)}\left[T_{X}(p)\right]\right] \\
& =\sum_{\gamma_{X}^{\subset} M_{+}} T_{X}(\gamma) \otimes T_{X}\left(M_{+} / \gamma\right) .
\end{aligned}
$$

Now we have to take care of the difference between $T_{X}(\gamma)$ and $T_{\gamma}$, and between $T_{X}\left(M_{+} / \gamma\right)$ and $T_{M_{+} / \gamma}$.

We first take care of all possible differences between $T_{X}(\gamma)$ and $T_{\gamma}$. Consider all $\gamma \in \mathcal{P}_{X}\left(M_{+}\right)$. First, we consider all such $\gamma$ which are in $\mathcal{M}^{[2]}$ and overlapping. In the coproduct (18) we find a term $T_{X}(\gamma)$ on the lhs. $T_{X}(\gamma) \otimes T_{X}\left(M_{+} / \gamma\right)$ is actually a sum of terms (as on both sides are sums of trees in general) which carries a natural product structure indicated by the tensorproduct. For each term in this sum, there is a well-defined set of edges corresponding to the admissible cut which gives $\gamma$. Gluing both sides, $T_{X}(\gamma)$ and $T_{X}(M / \gamma)$, together along these edges gives back $T_{X}\left(M_{+}\right)$,

$$
T_{X}(M)=T_{X}(\gamma) \wedge_{\gamma} T_{X}(M / \gamma)
$$

Here $\wedge_{\gamma}$ refers to the gluing process along the edges which are cut when we obtain $T_{X}(\gamma)$ on the lhs of the tensorproduct. Instead, we glue $T_{\gamma}=T_{X}(\gamma)+U_{\gamma}$ back along these edges ('surgery along edges'), for all overlapping $\gamma \in \mathcal{M}^{[2]}$. Call the new sum of trees

$$
T_{2}\left(M_{+}\right)=T_{\gamma} \wedge_{\gamma} T_{X}(M / \gamma)
$$

It has the form $T_{X}\left(M_{+}\right)+T_{2}$, where $n_{v}\left(T_{2}\right)=n_{v}\left(T_{X}\left(M_{+}\right)\right)-1$.

It further has the property that all cuts corresponding to such a $\gamma$ in $T_{2}\left(M_{+}\right)$ will give $T_{\gamma}$ on the lhs, if we employ $\Delta_{2}\left[T_{2}\left(M_{+}\right)\right]$. 
Now we consider all $\gamma \in \mathcal{P}_{X}\left(M_{+}\right)$which are overlapping and in $\mathcal{M}^{[3]}$. We use the product structure of $T_{2}(M)$ under $\Delta_{2}$ and glue back $T_{\gamma}$ for $T_{2}(\gamma)$. We continue in this manner for all overlapping $\gamma \in \mathcal{P}_{X}\left(M_{+}\right)$in ascending order until we reach $\gamma \in \mathcal{M}^{[k]}$. Call the resulting sum of trees $T_{k}(M)$.

In a similar manner, we then replace $T_{k}(M / \gamma)$ by $T_{M / \gamma}$ starting with $M / \gamma \in$ $\mathcal{M}^{[2]}$. We finally obtain a sum of trees $\breve{T}(M)=T_{X}(M)+$ terms of lower depth.

By construction, $\Delta_{k}\left(\breve{T}(M)-T_{X}(M)\right)$ contains all the terms which distinguish $\sum_{\gamma}{ }_{X}{ }_{M} T_{\gamma} \otimes T_{M / \gamma}$ from $\Delta\left(T_{X}(M)\right)$. Notably, $\Delta_{k}$ acts on $\breve{T}(M)$, as it is a sum of rooted trees with decorations in $\mathcal{M}^{[1]} \cup_{i=2}^{k} U^{[i]}$.

Hence we get

$$
\Delta_{k}(\breve{T}(M))=\breve{T}(M) \otimes e+e \otimes \breve{T}(M)+\sum_{\gamma_{X} M} T_{\gamma} \otimes T_{M / \gamma}
$$

We now set

$$
\Delta_{k+1}\left(T_{M}\right):=T_{M} \otimes e+e \otimes T_{M}+(i d-\bar{e}) \otimes(i d-\bar{e}) \Delta_{k}(\breve{T}(M)) .
$$

Then, again, $U_{M}:=T_{M}-\breve{T}(M)$ is a primitive element for $\Delta_{k+1}$, and thus $\Delta_{k+1}$ becomes the coproduct of a Hopf algebra of rooted trees $\mathcal{H}_{R}\left(\mathcal{M}^{[1]} \cup_{i=2}^{k+1} U^{[i]}\right)$, where $U^{[k+1]}$ is the set of elements $U_{M}$, with $M$ an overlapping set of depth $k+1$, thus in $\mathcal{M}^{[k+1]}$. We have, in analogy to the case $k=2$,

$$
U^{[k+1]}=\left\{u_{M} \mid t_{1}\left(u_{M}\right)=T_{M}-\breve{T}(M), M \in \mathcal{M}^{[k+1]} \wedge M \notin \mathcal{M}^{n o l}\right\},
$$

which works iteratively as $\breve{T}$ uses only decorations obtained from trees with degree $\leq k$.

Hence, algorithmically, one needs to determine all elements $\gamma \in \mathcal{P}_{X}(M)$, and then all elements in the corresponding $\mathcal{P}_{X}(\gamma)$, and so on. Eventually, one ends considering elements of depth two, whose decorations can be immediately determined, by (17). One then works up with the grading.

We conclude that the natural coproduct (13) is the coproduct of the Hopf algebra of rooted trees based on an appropriate set of decorations, constructed iteratively starting at depth two and using induction on the grading by depth.

Regarding an arbitrary Feynman graph as a set of edges and vertices, it needs powercounting and a determination of (one-particle irreducible) subgraphs to determine the skeleton expansion, and hence all decorations, iteratively, as the examples in the next section will exhibit.

\section{Overlapping Divergences}

We will apply the notions established in the previous section to sets of propagators and vertices which constitute Feynman graphs. We will order Feynman graphs by the depth of the rooted trees assigned to them. Below, we will define 
an orderly condition $X$ which can be tested by powercounting. The elementary fact that Feynman integrals allow for a well-defined degree of divergence essentially allows to use this degree of divergence as the crucial check on subgraphs, regarded as subsets of edges and vertices. One-particle irreducibility is the other demand which we choose for convenience.

For each one-particle irreducible (1PI) superficially divergent Feynman graph $\Gamma$ we denote by $\{\Gamma\}$ the set of its propagators and vertices.

Let $X$ be the condition: for any set $\{\gamma\} \subset\{\Gamma\}$ of propagators and vertices $\mathbf{X}(\{\gamma\})$ is true if and only if $\gamma$ constitutes a one-particle irreducible superficially divergent subgraph of $\Gamma$.

Further, to $\mathbf{X}(\{\Gamma\} /\{\gamma\})$ we associate the graph $\Gamma / \gamma$ which we obtain if we shrink $\gamma$ in $\Gamma$ to a point.

Prop.5 $X$ is an orderly condition.

Proof: $\#\left(P_{X}(\{\Gamma\} /\{\gamma\})\right)=\# P_{X_{\{\gamma\}}}(M)$. Assume that two elements of either of these two sets correspond to the same two subgraphs of $\Gamma$. Then, if they are overlapping, nested or disjoint in one of these two sets, they are so in the other as well.

We are interested in the set $\mathcal{P}_{X}(\{\Gamma\})$. For a 1PI Feynman graph $\Gamma, T_{X}(\{\Gamma\})$ is the forest assigned to it in the sense of the previous section. In general, $T_{X}(\{\Gamma\})$ will be a sum of rooted trees $T_{X}(p), p \in \mathcal{P}_{X}^{\text {cit }}(\{\Gamma\})$. Note further that $\{\Gamma\}$ is irreducible with respect to $X$ for all 1PI graphs $\Gamma$.

Define the depth $d(\Gamma)$ as

$$
d(\Gamma):=n_{v}(T(\{\Gamma\})),
$$

as before. This depth is well-defined for any Feynman graph.

Feynman diagrams without subdivergences thus have depth one, as they correspond to the rooted tree $t_{1}$ decorated by the set $\{\Gamma\}$.

Each Feynman diagram has a well-defined depth and thus we have a decomposition on the set of all Feynman graphs $\mathcal{F} \mathcal{G}$,

$$
\mathcal{F G}=\mathcal{F} \mathcal{G}^{[0]} \cup \mathcal{F} \mathcal{G}^{[1]} \cup \mathcal{F} \mathcal{G}^{[2]} \cup \mathcal{F} \mathcal{G}^{[3]} \cup \ldots
$$

Here $\mathcal{F} \mathcal{G}^{[0]}$ corresponds to superficially convergent graphs. We are interested in graphs in $\mathcal{F} \mathcal{G}^{[n]}, n \geq 1$.

To Feynman graphs of depth one we assign the rooted tree $t_{1}$, decorated by the corresponding element of $\mathcal{F} \mathcal{G}^{[1]}$. The elements of this set furnish the set of primitive elements of the Hopf algebra $\mathcal{H}_{R}\left(\mathcal{F G}^{[1]}\right)$ of decorated rooted trees.

The results of the previous section show that for each Feynman graph $\Gamma \in$ $\mathcal{F} \mathcal{G}^{[k]}$, we find a sum of associated rooted tree $T_{\Gamma}$ and a coproduct given by

$$
\Delta\left(T_{\Gamma}\right)=1 \otimes T_{\Gamma}+T_{\Gamma} \otimes 1+\sum_{\gamma}{ }_{X} \Gamma
$$


Here, $T_{\Gamma}$ is a sum of rooted trees with decorations in $\mathcal{F} \mathcal{G}^{[1]}$ and in $\cup_{i=2}^{k} U^{[i]}$, primitive elements in the Hopf algebra of rooted trees, obtained from Feynman graphs without subdivergences (which, as said earlier in this paper, includes graphs which have other subgraphs reduced to a point in them) and iteratively constructed primitive elements in $U^{[i]}$ as described in the previous section.

We will soon see explicit examples which indeed show that the so constructed elements are indeed primitive, hence correspond to analytic expressions without subdivergences.

At this stage, we can justify the notation of [2] or [4], where vertices of rooted trees where decorated by elements of $\mathcal{F} \mathcal{G}^{[1]}[$ [1] , which in the same spirit were used as letters of parenthesized words in [2]. In Prop. 2 we labelled each vertex $v$ of $T(\{\Gamma\})$ by a subset $\{\gamma\}$ corresponding to a subgraph $\gamma$ in our context. $\gamma$ itself can have further subdivergences. But then, condition $X$ and Prop. 2 ensure that we could as well label vertices by elements of $\gamma(v) / \gamma_{v}$, which correspond to graphs without subdivergences.

Before we come to examples, let us first make sure that we really get Zimmermann's forest formula from (20).

\subsection{Derivation of the forest formula}

To the coproduct (20) belongs an antipode given by

$$
S\left(T_{\Gamma}\right)=-T_{\Gamma}-\sum_{\gamma \subset \Gamma} S\left[T_{\gamma}\right] T_{\Gamma / \gamma}
$$

as one immediately checks. As it is an antipode in a Hopf algebra of rooted trees, it can be written as a sum over all cuts. Set $T_{\Gamma}=\sum_{i} T_{i}$ for some decorated rooted trees $T_{i}$. Then,

$$
S\left(T_{\Gamma}\right)=\sum_{i} \sum_{\text {all cuts } C_{i} \text { of } T_{i}}(-1)^{n_{C_{i}}} P^{C_{i}}\left(T_{i}\right) R^{C_{i}}\left(T_{i}\right) .
$$

Each such cut corresponds to a renormalization forest, which we obtain if we box the corresponding subgraphs in $\Gamma$, and vice versa [4]. 3]

Now, let $\phi$ be a $\mathbf{Q}$-linear map which assigns to $T_{\Gamma}$ the corresponding Feynman integral. Further, let $\phi_{R}=\tau_{R} \circ \phi$ be a map which assigns to $T_{\Gamma}$ the corresponding Feynman integral, evaluated under some renormalization condition $R$. Hence, from $T_{\Gamma}$ we obtain via $\phi$ a Feynman integral $\phi\left(T_{\Gamma}\right)$ in need of renormalization. $\tau_{R}$ modifies this Feynman integral, in a way such that the result contains the divergent part of this integral. Essentially, $\tau_{R}$ extracts the divergences of $\phi\left(T_{\Gamma}\right)$ in a meaningful way [6]. Hence, as $\tau_{R}$ isolates divergences faithfully, differences $\left(i d-\tau_{R}\right)\left(\phi\left(T_{\Gamma}\right)\right)$ eliminate divergences in Feynman integrals. Depending on the chosen renormalization scheme $R$, one can adjust finite

\footnotetext{
${ }^{3}$ Note that we can easily identify maximal forests here (in the sense of renormalization theory), by using the $B_{-}$operator on the trees $T_{i}$.
} 
parts to fulfil renormalization conditions. A detailed study of this freedom from the Hopf algebra viewpoint can be found in [9].

We remind the reader of Sweedler's notation: $\Delta\left(T_{\Gamma}\right)=\sum T_{\Gamma(1)} \otimes T_{\Gamma(2)}$. Let us consider the antipode $\bar{e}\left(T_{\Gamma}\right)$ using Sweedler's notation:

$$
0=\bar{e}\left(T_{\Gamma}\right)=\sum S\left(T_{\Gamma(1)}\right) T_{\Gamma(2)} .
$$

This map vanishes identically. Note that it can also be written as

$$
m\left[(S \otimes i d) \Delta\left(T_{\Gamma}\right)\right] \equiv \bar{e}\left(T_{\Gamma}\right)=0 .
$$

But this map gives rise to a much more interesting map, by composition with $\phi$,

$$
T_{\Gamma} \rightarrow \Gamma_{R}:=m\left[\left(S_{R} \otimes i d\right)(\phi \otimes \phi) \Delta\left(T_{\Gamma}\right)\right] .
$$

This map associates to the Feynman graph $\Gamma$ represented by a unique sum of rooted trees the renormalized Feynman integral $\Gamma_{R}$ 迆, 团.

Its usual definition

$$
\Gamma_{R}=\left(i d-\tau_{R}\right)\left[\Gamma+\sum_{\gamma \subset \Gamma} Z_{\gamma} \Gamma / \gamma\right],
$$

is recovered if we define

$$
S_{R}\left[\phi\left(T_{\gamma}\right)\right] \equiv Z_{\gamma}=-\tau_{R}(\gamma)-\tau_{R}\left[\sum_{\gamma^{\prime} \subset \gamma} Z_{\gamma^{\prime}} \gamma / \gamma^{\prime}\right] .
$$

This map is derived from the antipode

$$
S\left[T_{\gamma}\right]=-T_{\gamma}-\sum_{\gamma^{\prime} \subset \gamma} S\left[T_{\gamma^{\prime}}\right] T_{\gamma / \gamma^{\prime}}
$$

Using $\phi$ to lift this to Feynman graphs, and using the freedom to alter corresponding analytic expressions according to renormalization schemes $R$ one obtains (24).

Note that if one defines

$$
\phi_{R}=S_{R} \circ \phi \circ S,
$$

one has $S_{R} \circ \phi=\phi_{R} \circ S$ and hence

$$
S_{R}\left[\phi\left(T_{\gamma}\right)\right]=\tau_{R}\left[-\phi\left(T_{\gamma}\right)-\sum_{\gamma^{\prime} \subset \gamma} \phi_{R}\left(S\left[T_{\gamma^{\prime}}\right]\right) \phi\left(T_{\gamma / \gamma^{\prime}}\right) .\right]
$$

Hence, in accordance with [2, 4] we find the $Z$-factor of a graph $\gamma$ as derived from the antipode in the Hopf algebra of rooted trees. Above, in (23), we 
recovered the original forest formula in its recursive form. The non-recursive form is recovered with the same ease, using (22) instead of (21) [2, 4. It reads

$$
\Gamma_{R}=\left(i d-\tau_{R}\right)\left[\sum_{i} \sum_{\text {all normal cuts }}(-1)^{n_{C_{i}}} \phi_{\tau_{R}}\left(P^{C_{i}}\left(T_{i}\right)\right) \phi\left(R^{C_{i}}\left(T_{i}\right)\right)\right]
$$

in a form which makes its finiteness obvious when we take into account that the operation $\tau_{R}$ is defined to leave divergences unaltered. $\phi_{\tau_{R}}\left(P^{C_{i}}\left(T_{i}\right)\right)$ implies an iterative application of $\tau_{R}$ as governed by the unique boxes (the forests of classical renormalization theory) associated with normal cuts (4. Explicit realizations will be given elsewhere [9], as well as a more detailed discussion of renormalization schemes, renormalization group equations, operator product expansions and relations to cohomological properties of renormalizations.

\subsection{Examples}

We start with a simple example. Let $v_{1}, v_{2}, \omega_{1}, \omega_{2}, \omega_{3}$ be the Feynman graphs indicated in Fig. (7). We then have, switching to a notation in PW's [2], ${ }^{4}$

$$
\begin{aligned}
T_{X}\left(\omega_{3}\right) & =\left(\left(v_{1}\right) \omega_{1}\right)+\left(\left(v_{2}\right) \omega_{2}\right), \\
T_{\omega_{3}} & =\left(\left(v_{1}\right) \omega_{1}\right)+\left(\left(v_{2}\right) \omega_{2}\right)+\left(U_{\omega_{3}}\right), \\
\left(U_{\omega_{3}}\right) & =\left(T_{\omega_{3}}\right)-\left[\left(\left(v_{1}\right) \omega_{1}\right)+\left(\left(v_{2}\right) \omega_{2}\right)\right], \\
\Delta\left[T_{\omega_{3}}\right] & =T_{\omega_{3}} \otimes e+e \otimes T_{\omega_{3}}+\left(v_{1}\right) \otimes\left(\omega_{1}\right)+\left(v_{2}\right) \otimes \omega_{2} .
\end{aligned}
$$

The graphs belong to $\mathcal{F} \mathcal{G}^{[2]}$.

Note that $U_{\omega_{3}}$ gives us the skeleton corresponding to this graph. It is a primitive element, and thus free of subdivergences. And indeed, for any choice of momentum transfer and masses in $v_{i}, \phi\left(U_{\omega_{3}}\right)$ is an analytic expression free of subdivergences. Any representation of $T_{\Gamma}$ in terms of Feynman integrals shows that the expressions corresponding to such $U_{\Gamma}$ are free of subdivergences. An instructive example is given in the appendix of [1], where it is shown how graphs in $\phi^{3}$ theory explicitly realize the results derived here on general grounds. Similar results can be found in [2, 3, 7].

Next, in Fig.(8), we consider examples taken from $\mathcal{F} \mathcal{G}^{[3]}$.

This time, we find the following results

$$
\begin{aligned}
T_{X}\left(\omega_{4}\right)= & \left(\left(\left(v_{2}\right) v_{3}\right) \omega_{2}\right)+\left(\left(\left(v_{3}\right) v_{1}\right) \omega_{1}\right)+\left(\left(v_{3}\right)\left(v_{2}\right) \omega_{2}\right), \\
\breve{T}\left(\omega_{4}\right)= & \left(\left(v_{2}\right) U_{\omega_{5}}\right)+\left(\left(v_{3}\right) U_{\omega_{3}}\right), \\
T_{\omega_{4}}= & \left(\left(\left(v_{2}\right) v_{3}\right) \omega_{2}\right)+\left(\left(\left(v_{3}\right) v_{1}\right) \omega_{1}\right)+\left(\left(v_{3}\right)\left(v_{2}\right) \omega_{2}\right) \\
& +\left(\left(v_{2}\right) U_{\omega_{5}}\right)+\left(\left(v_{3}\right) U_{\omega_{3}}\right)+U_{\omega_{4}} .
\end{aligned}
$$

\footnotetext{
${ }^{4}$ For example, in this notation $\left(\left(v_{1}\right) \omega_{1}\right)$ corresponds to the tree $t_{2}$, with its root decorated by $\omega_{1}$ and the other vertex decorated by $v_{1}$. Decorated rooted trees and PW's on an alphabet of decorations are in one-to-one correspondence th.
} 


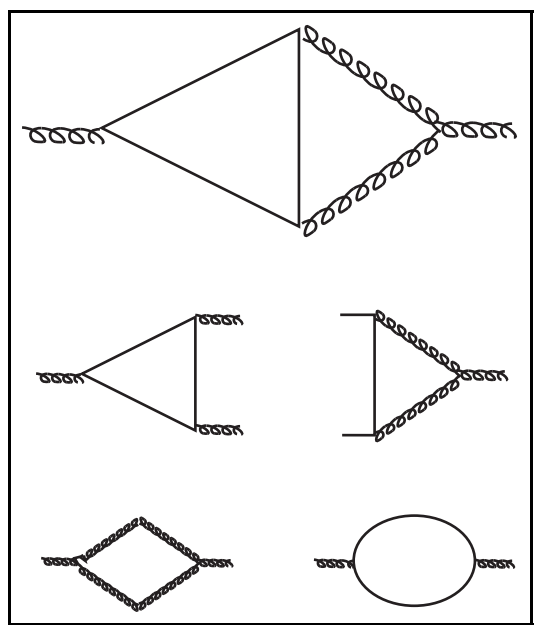

Figure 7: A graph from $\mathcal{F} \mathcal{G}^{[2]}$ and its subgraphs. We read it as a graph in Yang-Mills theory in four dimensions say, with straight lines being fermions. In the first row, we see the graph $\omega_{3}$. Below, we see its two subgraphs $v_{1}, v_{2}$ and in the bottom row we see the graphs $\omega_{1}=\omega_{3} / v_{1}$ and $\omega_{2}=\omega_{3} / v_{2}$.

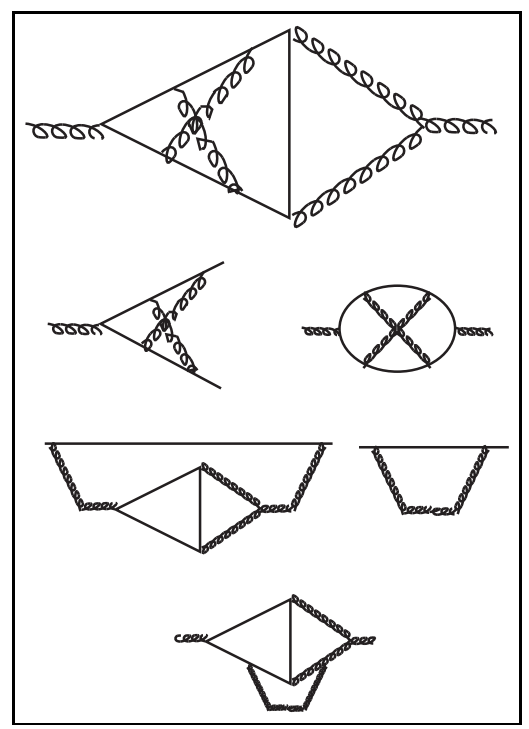

Figure 8: Graphs from $\mathcal{F} \mathcal{G}^{[3]}$ and their subgraphs. At the top, we see the graph $\omega_{4}$. Apart from the subgraphs in the previous figure, we find two more subgraphs, the vertex $v_{3}$ and the self-energy $\omega_{5}=\omega_{4} / v_{2}$, both given in the second row. In the third row, we define the three-loop fermion self-energy $\Sigma_{3}$. It involves the same subgraphs as before, plus a new graph $\Sigma_{1}=\Sigma_{3} / \omega_{3}$. Finally, at the bottom, we see the graph $\omega_{6}$. 


$$
\begin{aligned}
\Delta\left(T_{\omega_{4}}\right)= & T_{\omega_{4}} \otimes e+e \otimes T_{\omega_{4}} \\
& +2\left(v_{2}\right) \otimes\left(\left(v_{3}\right) \omega_{2}\right)+\left(v_{3}\right) \otimes\left(\left(v_{1}\right) \omega_{1}\right)+\left(v_{3}\right) \otimes\left(\left(v_{2}\right) \omega_{2}\right) \\
& +\left(\left(v_{2}\right) v_{3}\right) \otimes\left(\omega_{2}\right)+\left(\left(v_{3}\right) v_{1}\right) \otimes\left(\omega_{1}\right)+\left(v_{3}\right)\left(v_{2}\right) \otimes\left(\omega_{2}\right) \\
& +\left(v_{2}\right) \otimes\left(U_{\omega_{5}}\right)+\left(v_{3}\right) \otimes\left(U_{\omega_{3}}\right) .
\end{aligned}
$$

Now,

$$
\begin{aligned}
& 2\left(v_{2}\right) \otimes\left(\left(v_{3}\right) \omega_{2}\right)+\left(v_{3}\right) \otimes\left(\left(v_{1}\right) \omega_{1}\right)+\left(v_{3}\right) \otimes\left(\left(v_{2}\right) \omega_{2}\right) \\
& +\left(v_{2}\right) \otimes\left(U_{\omega_{5}}\right)+\left(v_{3}\right) \otimes\left(U_{\omega_{3}}\right) \\
& =\left(v_{2}\right) \otimes T_{\omega_{5}}+\left(v_{3}\right) \otimes T_{\omega_{3}},
\end{aligned}
$$

as

$$
\begin{aligned}
& T_{\omega_{5}}=U_{\omega_{5}}-2\left(\left(v_{3}\right) \omega_{2}\right), \\
& T_{\omega_{3}}=U_{\omega_{3}}-\left(\left(v_{2}\right) \omega_{2}\right)-\left(\left(v_{1}\right) \omega_{1}\right) .
\end{aligned}
$$

For the other graphs in Fig.(8) we find

$$
T_{\Sigma_{3}}=\left(\left(\left(v_{1}\right) \omega_{1}\right) \Sigma_{1}\right)+\left(\left(\left(v_{2}\right) \omega_{2}\right) \Sigma_{1}\right)+\left(\left(U_{\omega_{3}}\right) \Sigma_{1}\right),
$$

and

$$
T_{\omega_{6}}=\left(\left(\left(v_{2}\right) v_{1}\right) \omega_{1}\right)+\left(\left(\left(v_{2}\right) v_{2}\right) \omega_{2}\right)+\left(\left(v_{2}\right) U_{\omega_{3}}\right) .
$$

We invite the reader to confirm that the coproduct on these expressions has the desired form (13).

Finally, Figs. (9, 10, 11) shows how the transition from $T_{X}(\Gamma)$ to $T_{\Gamma}$ is achieved in terms of surgery along edges. We start with an example taken from $\phi^{3}$ theory in six dimensions. We consider a quadratically divergent two-point function as given in the figures. Fig.(9) gives $T_{X}(\Gamma)$. It consists of six decorated rooted trees. In the figure, we give the decorations not by primitive elements, but by full subgraphs. The decoration by primitive elements is obtained, in accordance with Prop.2, if we divide by the decorations at outgoing vertices. That there are six decorated trees if a consequence of the internal product structure of the graph: there is a subgraph $\gamma_{2}$ with $\#\left(\mathcal{P}_{X}^{\text {cit }}\left(\gamma_{2}\right)\right)=2$, and the complement graph $\Gamma / \gamma_{2}$ has $\#\left(\mathcal{P}_{x}^{\text {cit }}\left(\Gamma / \gamma_{2}\right)\right)=3$.

Fig.(10) adds the terms for the transition $T_{X}(\gamma) \rightarrow T_{\gamma}$. This is only nontrivial for the case that $\gamma$ is the indicated overlapping two-loop two-point function $\gamma_{2}$. Finally, Fig.(11) shows the additional terms generated from the complement graphs $\Gamma / \gamma$.

Let us end this section with a few remarks concerning the various sorts of overlapping divergences. Most prominent and most severe are overlapping quadratic divergences, as one encounters typically in (gauge)-boson propagators, let it be gauge theory in four dimensions of $\phi^{3}$ theory in six, considered in the above examples. Typically, the overlapping subdivergences are provided by 


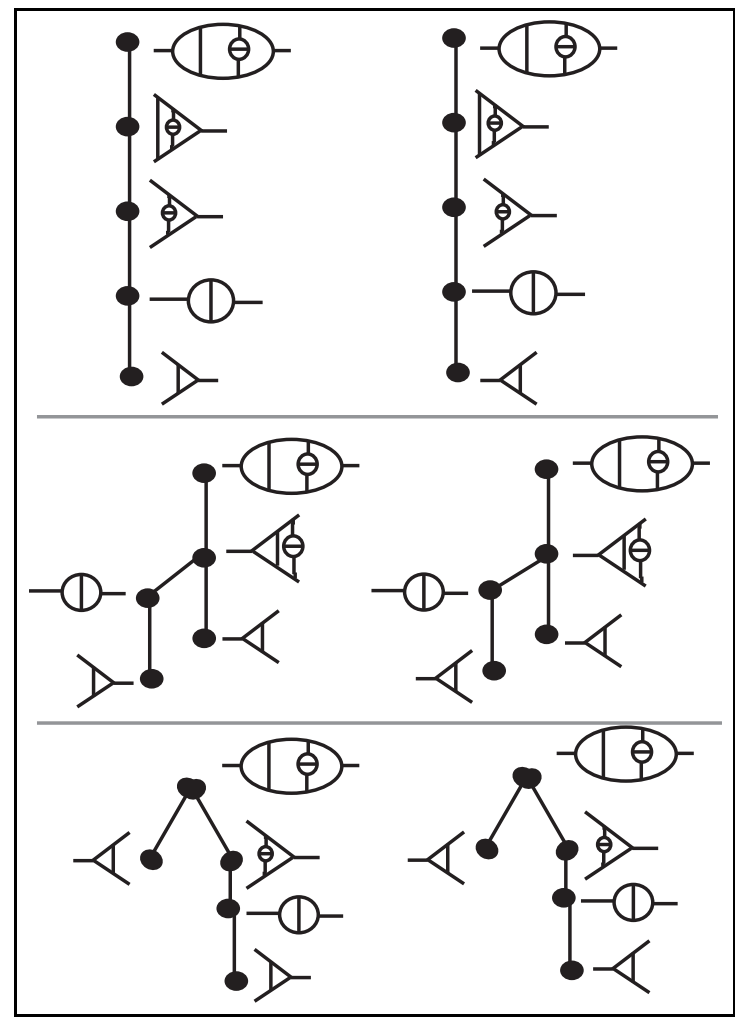

Figure 9: Surgery along edges delivers the transition from $T_{X}(\Gamma)$ to $T_{\Gamma}$. We first give $T_{X}(\Gamma)$, where $\Gamma$ is the five-loop graph indicated at the roots. All six rooted trees in this figure have to be added to give $T_{X}(\Gamma)$. 


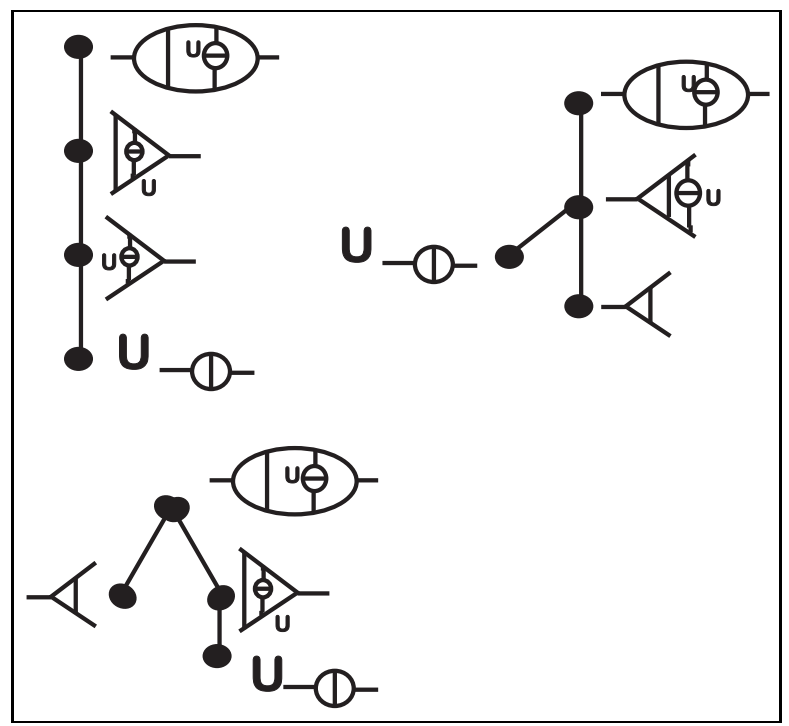

Figure 10: Now we add the results of replacing $T(\gamma)$ by $T_{\gamma}$.

vertex corrections, and hence we have two sets which overlap. Characteristically, the two overlapping subdivergences can be eleminated by two derivatives with respect to an external momentum, one for each of them. This then generates new decorations of logarithmic degree of divergence. An illuminating example for this situation is given in the appendix of 仼.

Overlapping degrees of divergences can come in other degrees of divergence, and in other configurations. For example, in non-abelian Yang-Mills theory one can have overlapping divergent Feynman graphs with a logarithmic degree of divergence, where one has three sets which mutually overlap with each other.

\section{Conclusions}

Starting from set-theoretic notions, we showed how the forest formula underlying renormalization theory is ad initio derived from the Hopf algebra of rooted trees. At the same time, we constructed a systematic way how to obtain the skeleton expansion in any QFT, given by elements $U_{M}$. We derived the original nonrecursive forest formula of Zimmermann from the Hopf algebra of rooted trees, as well as the recursive formulation. The results of [5] are in full accordance with our results and are a specification of the general result presented here.

\footnotetext{
${ }^{5} \mathrm{~A}$ tetrahedron formed out of gluons, with three external gluons coupling to three sides of the tetrahedron which form a triangle is an appealing three-loop example only involving three-gauge-boson vertices.
} 


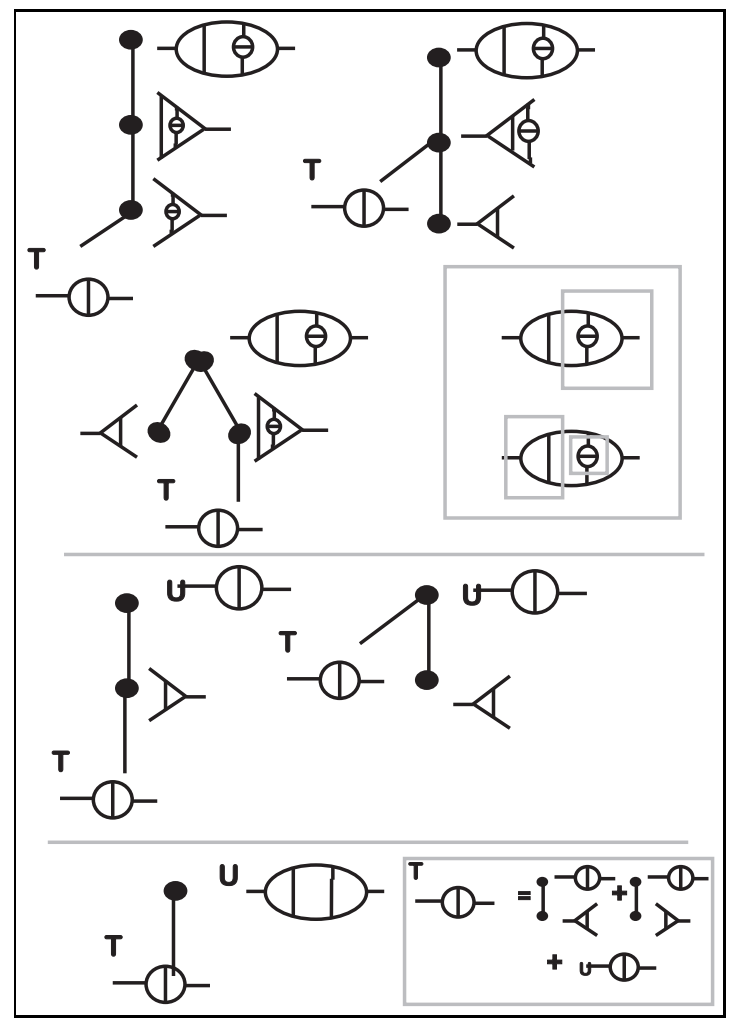

Figure 11: Finally, we construct the terms which achieve the transition $T_{X}(\Gamma / \gamma) \rightarrow$ $T_{\Gamma / \gamma}$. The first two rows, if we append the forest $T_{\gamma}$, give the terms of the previous two figures. The second takes into account the fact that in $\Gamma / \gamma, \forall \gamma \in \mathcal{P}_{X}(\{\Gamma\})$, we can find the element $\gamma_{2}=\Gamma / \gamma$ itself, by shrinking three loops to this element of $\mathcal{F} \mathcal{G}^{[2]}$. The inlay in the first row indicates the graphs $\gamma$ which have to shrink. Note that $\gamma$ is allowed to consist of disjoint graphs. The last row takes into account the primitive element $U_{\Gamma / \gamma_{2}}$. The inlay defines $T_{\gamma_{2}}$. 
Details for the practitioner of calculational QFT are given elsewhere [9], including remarkable number-theoretic results when investigating the role of the Connes-Moscovici Hopf subalgebra in Feynman diagrams.

Some further remarks are in order.

- The methods developped in the first section are sufficiently general to be applied to problems of operator product expansions and asymptotic expansions, with applications to OPE's already being established [9]. Our approach being based on set-theoretic considerations, the remaining challenge for general asymptotic expansions is to find and interpret sensible conditions $X$, and to identify the resulting primitive elements.

- The Hopf algebra of rooted trees has relations to shuffle Hopf algebras 8. Shuffle products play a role when we start to study the action of the symmetric group on decorations. They appear naturally in the consideration of the sub Hopf algebra generated by trees $B_{+}^{k}(e)$, which is the Hopf algebra underlying Chen's iterated integral. The Hopf algebra of rooted trees has this algebra as a sub Hopf algebra. There are interesting generalizations when we study shuffle algebras and iterated integrals from the viewpoint of the Hopf algebra of rooted trees [9]. Especially, the absence of a shuffle product for bare Green functions in the presence of a remaining convolution law points to interesting structures lying ahead [9].

- The general set-theoretic set-up adopted in this paper allows to study bare Green functions in $x$-space, and hence will allow to study them as functions on configuration space (which relies on tree-ordered boundaries in a natural manner, see e.g.[10] and references there). This will hopefully reconcile early work on such functions [1] with more recent developments.

\section{Acknowledgements}

Let me first thank Raymond Stora for interest and discussions, and for the ultimate motivation to write this paper. Also, I thank him for carefully proofreading an earlier version of this paper. I very much enjoyed the opportunity to discuss the intricate structures of the calculus of QFT and to collaborate on its surprising relation to Noncommutative Geometry with Alain Connes. I also thank Alain for generous hospitality on various occasions. As usual, many thanks are due to David Broadhurst for companionship in our longlasting exploration of patterns and structures in QFT. Support by a Heisenberg fellowship is gratefully acknowledged.

\section{References}

[1] W. Zimmermann, Comm.Math.Phys.15 (1969) 208. 
[2] D. Kreimer, Adv.Theor.Math.Phys.2, 303 (1998); q-alg/9707029.

[3] D. Kreimer, J.Knot Th.Ram.6 479 (1997); q-alg/9607022.

[4] A. Connes, D. Kreimer, Comm.Math.Phys.199 203 (1998); hepth/9808042.

[5] T. Krajewski, R. Wulkenhaar, On Kreimer's Hopf algebra structure of Feynman Graphs, CPT-98/P.3639; hep-th/9805098, to appear in Eur.Phys.J.C.

[6] J.C. Collins, Renormalization, Cambridge Univ. Press (1984).

[7] D.J. Broadhurst, R. Delbourgo, D. Kreimer, Phys.Lett.B366 421 (1996); hep-ph/9509296.

[8] M.E. Hoffman, Quasi-shuffle products, preprint, to appear in J. Algebraic Combinatorics;

J.M. Borwein, D.M. Bradley, D.J. Broadhurst, P. Lisonek, Combinatorial aspects of multiple zeta values, Electr.J.Comb.5 (1998), R38.

[9] D.J. Broadhurst, D. Kreimer, Renormalization automated by Hopf algebras, hep-th/9810087,

D. Kreimer, Chen's iterated integral represents the Operator Product Expansion, hep-th/9901099;

R. Delbourgo, D. Kreimer, Using Hopf algebras to calculate Feynman diagrams, in preparation.

[10] D.P. Thurston, Integral Expressions for the Vassiliev Knot Invariants, math/9901110.

[11] H. Epstein, V. Glaser, R. Stora, General Properties of the n-point Functions in Local Quantum Field Theory, in Les Houches 1975, Proceedings, Summer School On Structural Analysis Of Collision Amplitudes, Amsterdam 1976, 5-93;

H. Epstein, V. Glaser, Ann. Inst. H. Poincaré19 211 (1973). 